\title{
Computed Tomography Density Change in the Thyroid Gland Before and After Radiation Therapy
}

\author{
NAOYA ISHIBASHI ${ }^{1}$, TOSHIYA MAEBAYASHI ${ }^{1}$, TAKUYA AIZAWA ${ }^{1}$, \\ MASAKUNI SAKAGUCHI ${ }^{1}$, MASAHIRO OKADA ${ }^{1}$ and JUNICHI MATSUSHITA ${ }^{2}$ \\ ${ }^{1}$ Department of Radiology, Nihon University School of Medicine, Tokyo, Japan; \\ ${ }^{2}$ Department of Radiological Technology, Nihon University School of Medicine, Tokyo, Japan
}

\begin{abstract}
Background/Aim: Hypothyroidism is an established adverse effect of radiation therapy for head and neck cancer, and computed tomography (CT) density of the thyroid gland is lower in hypothyroid than euthyroid individuals. No previous studies have evaluated changes in CT densities of the thyroid gland caused by radiation therapy. The aim was to investigate the relationship between the change in CT density of the thyroid gland before and after radiation therapy for head and neck cancer and hypothyroidism. Materials and Methods: This retrospective study analyzed data of 24 patients treated by radiation therapy for head and neck cancers. After dosimetric analysis of received radiation therapy, a Picture Archiving and Communication System was used to manually contour the thyroid on pre-treatment CT images to enable determination of mean thyroid gland $C T$ densities and received radiation doses. Pre-and post-treatment thyroid function was assessed on the basis of serum TSH concentrations. Multivariate and univariate analyses were used to determine what clinical factors are associated with post-radiation therapy decrease in CT density of the thyroid and Pearson's $\chi^{2}$ test was used to assess correlations between these densities and TSH concentrations. Results: Mean CT densities of the thyroid gland decreased from before to after radiation therapy in $73.9 \%$ of our patients (median decrease $16.8 \mathrm{HU}$ ). Serum TSH concentrations were significantly higher in patients with greater then median decreases in CT density than in those with lesser or no decreases. Conclusion: Post-radiation therapy hypothyroidism may be predicted by significant decreases in CT density of the thyroid gland.
\end{abstract}

Correspondence to: Dr. Naoya Ishibashi, Department of Radiology, Nihon University School of Medicine, 30-1, Oyaguchi Kami-cho Itabashi-ku, Tokyo 173-8610, Japan. Tel: +81 339728111, Fax: +81 339582454, e-mail: ishibashi.naoya@nihon-u.ac.jp

Key Words: CT density change, thyroid gland, radiation therapy, head and neck cancer, thyroid function.
Head and neck cancer is often treated by radiation therapy, the aim being to preserve thyroid function. Hypothyroidism is a well-known adverse effect of radiation therapy for head and neck cancer (1-3). It has also been established that the computed tomography (CT) density of the thyroid gland decreases in hypothyroidism caused by chronic thyroiditis and other conditions $(4,5)$. We therefore postulated that the onset of radiation therapy-induced hypothyroidism could be predicted on the basis of changes in CT densities of the thyroid gland before and after radiation therapy. No previous studies have provided substantial data on changes in $\mathrm{CT}$ densities of the thyroid gland caused by radiation therapy. In this study, we investigated the relationship between the change in CT densities of the thyroid gland before and after radiation therapy for head and neck cancer and hypothyroidism.

\section{Materials and Methods}

Patient characteristics. The study cohort comprised 24 patients who had started radiation therapy for biopsy-confirmed head and neck cancer between August 2012 and April 2017. This study was approved by the Institutional Review Board and the patients had given informed consent for treatment procedures.

Radiation therapy. All patients had received three-dimensional conformal radiation therapy (3D-CRT) with 4-MV photon beams on the right and left sides, the initial radiation field having included both the primary site gross tumor volume (GTV) and the involved or regional lymph node levels. Thus, all or part of the thyroid gland was included in the initial radiation field. The thyroid gland was not contoured on CT images taken for planning radiation therapy and the therapy was planned without treating the thyroid gland as an organ at risk. All patients received a radiation dose of $2 \mathrm{~Gy}$ once daily to a total dose of $66 \mathrm{~Gy}$.

Dosimetric analysis. After radiation therapy, the thyroid gland was manually contoured on the CT images that had been obtained to plan radiation therapy. Dose to the thyroid gland (minimum, maximum, and mean), percentage of thyroid volume that had received $>10,20,30,40,50$, and 60 Gy $(\mathrm{V} 10, \mathrm{~V} 20, \mathrm{~V} 30, \mathrm{~V} 40$, 
V50, and V60), and dose to $25 \%, 45 \%, 50 \%$, and $90 \%$ of the thyroid volume $\left(\mathrm{D}_{25}, \mathrm{D}_{45}, \mathrm{D}_{50}\right.$, and $\left.\mathrm{D}_{90}\right)$ were determined from dose-volume histograms (DVHs).

Computed tomography and measurement of CT densities. Diagnoses were made with a 320-slice multidetector CT scanner (Aquilion ONE; Toshiba Medical Systems, Tokyo, Japan) with a 4.7 -s scan time at $120 \mathrm{kV}$ and tube current (mA) automatically determined by the scanner using radiation dose modulation. Images were obtained using a soft tissue kernel at 1-mm thickness. A Picture Archiving and Communication System (Synapse Viewer; Fuji Medical Systems, Tokyo, Japan) was used to manually contour the thyroid glands on non-contrast CT image analysis, after which the mean CT densities of the thyroid glands (Hounsfield units, HU) were calculated using a three-dimensional image analysis device (Synapse Vincent; Fuji Medical Systems). The thyroid glands were carefully contoured while avoiding both artifacts resulting from calcification and cysts in the gland and the thyroid cartilage, cricoid cartilage, and clavicles. When CT densities had to be determined using images obtained with other CT models, differences between those images and those obtained with an Aquilion ONE were minimized by using several phantoms of CT densities of the thyroid gland and adjusting the densities to those determined on Aquilion ONE images. The CT images used to make diagnoses had been obtained a median of 24 days before the start of radiation therapy (range $=-4-1,400$ days) (in one patient images taken 4 days after beginning radiation therapy were regarded as taken before starting this treatment) and tumor responses assessment images a median of 359 days after completion of radiation therapy (range $=0-1,554$ days).

Evaluation of thyroid function. Thyroid function was assessed by measuring peripheral blood thyroid-stimulating hormone (TSH) concentrations before and after radiation therapy (normal range $=0.34-3.8 \mathrm{mIU} / \mathrm{L}$ ). These concentrations had been measured a median of 31 days before starting radiation therapy (range $=14-76$ days) and a median of 413 days after completing radiation therapy (range=7-1,352 days). Two patients for whom TSH concentrations before and after radiation therapy were not available were excluded from assessment of thyroid function.

Concurrent chemotherapy regimens. All patients had received systemic chemotherapy in combination with radiation therapy. The most common chemotherapy regimen comprised docetaxel, cisplatin, and 5-fluorouracil (TPF; 17 patients, 70.8\%), followed by a docetaxel-based regimen (five patients, 20.8\%) and cetuximab alone (two patients, $8.4 \%$ ).

Statistical analysis. SPSS version 21.0 (IBM, Armonk, NY, USA) was used for statistical analysis. Multivariate analysis using stepwise logistic regression and univariate analysis using Pearson's $\chi^{2}$ test and an independent sample $t$-test were performed to evaluate whether clinical factors, including age (<median $v s$. $\geq$ median), sex, chemotherapy regimen (TPF $v s$. others), and radiation therapy dosimetry (<median $v s$. $\geq$ median), were associated with decreases in CT densities of the thyroid gland from before to after radiation therapy. Moreover, decreases in CT densities and increases in TSH concentrations from before to after radiation therapy were evaluated using Pearson's $\chi^{2}$ test and frequency tables for the thyroid gland.
Table I. Patient, tumor and treatment characteristics.

\begin{tabular}{lc}
\hline Variables & No. of patients $(\%)$ \\
\hline Age (years) & Median 65 (Range=37-81 years) \\
Gender & $19(79.2)$ \\
Male & $5(20.8)$ \\
Female & \\
Tumor site & $2(8.3)$ \\
Nasopharynx & $8(33.4)$ \\
Oropharynx & $9(37.5)$ \\
Hypopharynx & $3(12.5)$ \\
Supraglottic & $2(8.3)$ \\
Oral cavity & \\
Clinical UICC stage & $2(8.3)$ \\
II & $7(29.2)$ \\
III & $15(62.5)$ \\
IV & $17(70.9)$ \\
Chemotherapy regimen & $5(20.8)$ \\
TPF & $2(8.3)$ \\
Docetaxel-based & \\
Cetuximab & \\
\hline
\end{tabular}

UICC: Union for International Cancer Control criteria 7th edition; TPF: docetaxel, cisplatin, and 5-fluorouracil.

\section{Results}

The characteristics of the 24 patients are summarized in Table I. They comprised of 19 men and 5 women of median age 65 years (range $=37-81$ years). The mean CT densities of the thyroid gland were $104.9 \pm 17.8 \mathrm{HU}$ (mean \pm standard deviation [SD]) before radiation therapy and 92.8 $\pm 24.0 \mathrm{HU}($ mean \pm SD) after radiation therapy. In one patient who had been treated for hypothyroidism with levothyroxine before radiation therapy, the mean CT density was $86.3 \mathrm{HU}$ and not the lowest in our patients, so this patient was included. CT densities decreased from before to after radiation therapy in 17 patients $(70.8 \%)$ (Figure 1). In these 17 patients, CT densities decreased by a median of 16.8 HU (range=1.9-48.6 HU). Thus, in this study a decrease of $\geq 16.8 \mathrm{HU}$ was defined as a significant decrease in CT density. Stepwise logistic regression analysis revealed the only one of these factors that correlated significantly with decrease in CT density was female sex $(p=0.050)$. Pearson's $\chi^{2}$ test also detected a significant correlation between female sex and decrease in CT density $(p=0.027)$. DVH analysis revealed that neither dose to the thyroid gland, nor percentage of thyroid volume irradiated or dose to thyroid volume correlated significantly with decreases in CT density.

Before radiation therapy, serum TSH concentrations exceeded $5 \mathrm{mIU} / \mathrm{L}$ in only one patient; this concentration indicates hypothyroidism according to the criteria of the Colorado Thyroid Disease Prevalence Study) (6) but this patient had not been treated for hypothyroidism so included. In another patient who had been treated for hypothyroidism with 
Table II. Changes in computed tomography (CT) densities and thyroid-stimulating hormone (TSH) concentrations before and after radiation therapy.

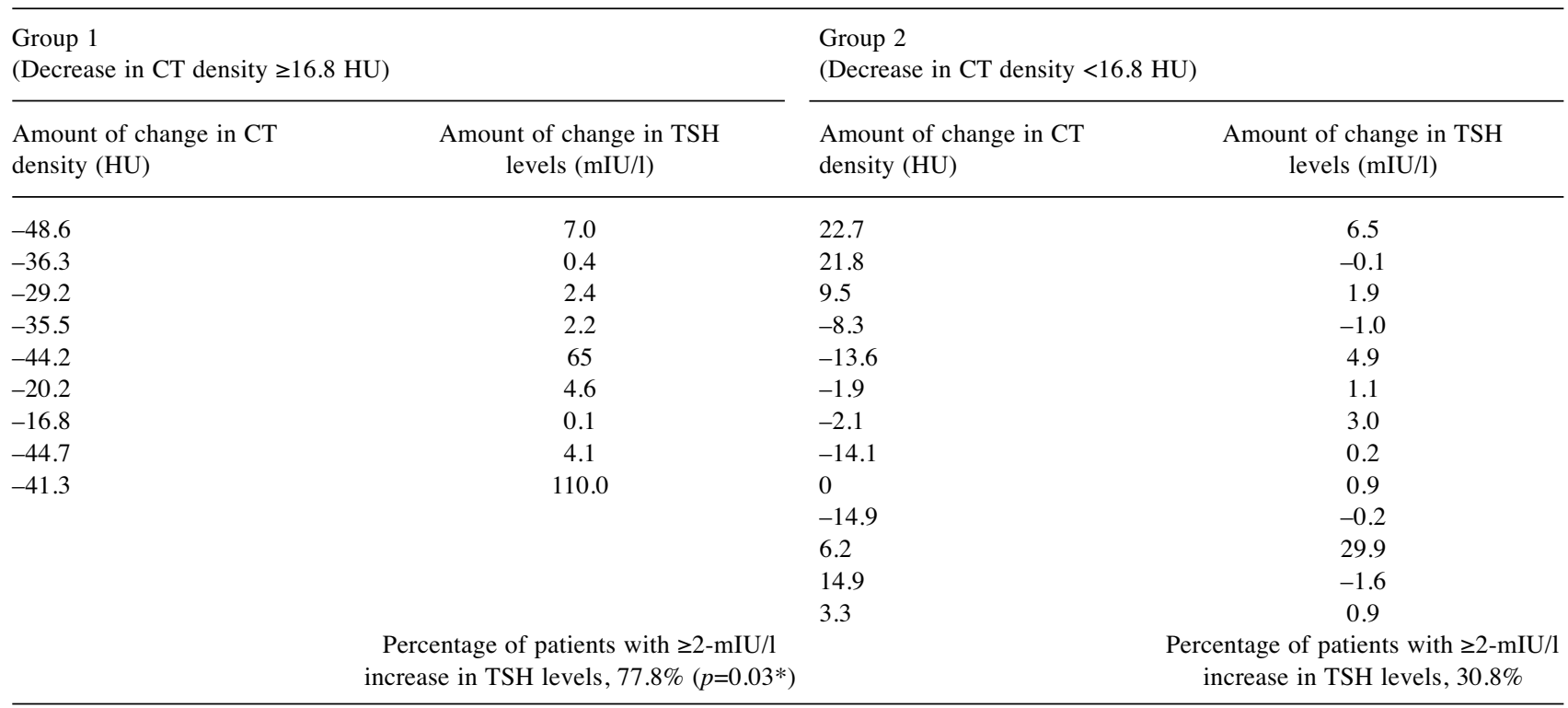

HU: Hounsfield units. ${ }^{*} \chi^{2}$ test.

levothyroxine before radiation therapy, TSH concentrations had been controlled within the normal range so this patient was included. Mean serum TSH concentrations were 2.4 $\pm 3.0 \mathrm{mIU} / \mathrm{L}$ (mean $\pm \mathrm{SD}$ ) before radiation therapy and $12.2 \pm 26.7 \mathrm{mIU} / \mathrm{L}$ $($ mean \pm SD) after radiation therapy. Serum TSH concentrations exceeded $5 \mathrm{mIU} / \mathrm{L}$ after radiation therapy in eight patients $(36.4 \%)$. From before to after radiation therapy, serum TSH concentrations increased in 18 patients $(81.8 \%)$ and decreased in four. No patient described a symptom associated with hypothyroidism. The median amount of change in serum TSH concentrations was $2.0 \mathrm{mIU} / \mathrm{L}$ (range $=-1.57-110.0 \mathrm{mIU} / \mathrm{L}$ ). Thus, in this study a significant increase in serum TSH concentrations was defined as an increase of $\geq 2.0 \mathrm{mIU} / \mathrm{L}$. Patients were allocated to two groups on the basis of changes in CT densities from before to after radiation therapy: Group 1 comprised nine patients in whom CT density had decreased by $\geq 16.8 \mathrm{HU}$ and Group 213 patients in whom CT density had decreased by $<16.8 \mathrm{HU}$. After radiation therapy, significant increases in serum TSH concentrations were observed in seven patients from Group 1 (77.8\%) and four from Group 2 (30.8\%). According to the $\chi^{2}$ test, there were more patients with significantly increased serum TSH concentrations in Group 1 than in Group $2(p=0.030)$ (Table II).

\section{Discussion}

The thyroid gland is often included in the radiation field when radiation therapy is administered for head and neck cancer. Hypothyroidism is a known late side-effect of

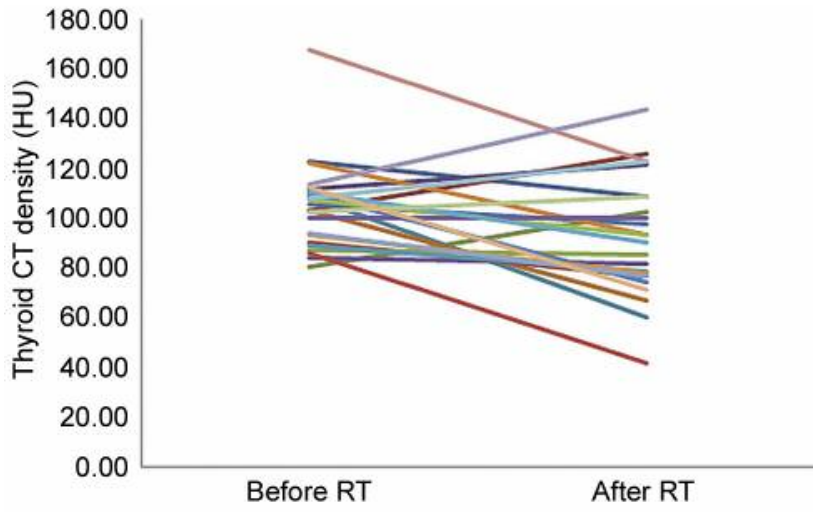

Figure 1. Computed tomography density changes of the thyroid gland before and after radiation therapy $(R T)$. HU: Hounsfield units.

radiation therapy, the reported incidence being between $10 \%$ and $50 \%(1-3,7-8)$. A diagnosis of hypothyroidism is generally based on an increased serum TSH concentration (1-3). In $36.4 \%$ of our patients, post-radiation therapy serum TSH concentrations exceeded $5 \mathrm{mIU} / \mathrm{L}$, which is the lower limit for diagnosing hypothyroidism (6). This proportion is comparable to previously reported findings in such patients.

It has since long been known that the thyroid gland is characterized by high attenuation density on non-contrast CT images (9). This is attributed to the fact that thyroid gland follicles contain iodine, which is an essential element for 
production of thyroid hormones. As iodine decreases, attenuation of CT density decreases $(9,10)$. Densities on noncontrast CT images of the thyroid gland have been visually assessed and classified as low and high attenuation (11). In this study, correlation of mean CT densities and thyroid function showed that a significantly greater proportion of patients with low than with high thyroid attenuation had hypothyroidism. Because iodine concentrations are controlled by a feedback loop involving serum TSH, densities of the thyroid gland on non-contrast CT images reportedly correlate with serum TSH concentrations $(4,5)$. For example, CT densities repeatedly decrease when TSH concentrations are $>3.0 \mathrm{mIU} / \mathrm{L}$ (5). In patients with normal serum $\mathrm{TSH}$ concentrations, mean CT densities of the thyroid gland on non-contrast CT images reportedly range from 97.4 to 122 $\mathrm{HU}(4,5)$. In our patients, the mean CT density of the thyroid gland before radiation therapy was $104.9 \mathrm{HU}$, which is comparable with previously reported densities in patients with normal thyroid function. In fact, serum TSH concentrations were $<5 \mathrm{mIU} / \mathrm{L}$ in all but one of our patients. Moreover, in patients with hypothyroidism caused by chronic thyroiditis and other conditions, mean CT densities of the thyroid gland on non-contrast images reportedly range between 81 and 82 HU $(4,5)$. In our patients, the mean CT density of the thyroid gland after radiation therapy was $92.8 \mathrm{HU}$, which is slightly higher than that previously reported in patients with hypothyroidism. This apparent discrepancy is attributable to the fact that our study included numerous patients with normal thyroid function, even after radiation therapy.

In our study, female sex was significantly correlated with a significant decrease in CT density from before to after radiation therapy. The fact that females are reportedly more likely than males to have permanent hypothyroidism after radiation therapy (3) is consistent with our finding that female sex is associated with decreases in CT density.

In recent years, various predictors of hypothyroidism attributable to radiation therapy have been identified by DVH analysis. For example, the mean radiation dose to the thyroid gland is one reported predictor $(2,12)$. The risk of developing hypothyroidism reportedly being higher when the mean radiation dose is $\geq 30$ Gy (2). Other reported predictors are V35-V50 values. In one study, the incidence of hypothyroidism was significantly higher when V45 was $\geq 50 \%$ (1). D25 to D50 are also predictors, the risk of developing hypothyroidism reportedly increasing when D50 is $\geq 50 \%$ (3). In the present study, we evaluated the association between the results of DVH analysis and changes in the CT densities of the thyroid gland before and after radiation therapy and found no significant correlation between the results of DVH analysis and decreases in the CT densities. Furthermore, not only radiation therapy, but also chemotherapy may cause hypothyroidism. Of the chemotherapeutic agents that our patients had received, fluoropyrimidine, reportedly has the potential to cause hypothyroidism in patients with colorectal cancer (13). We identified no significant correlations between various chemotherapy regimens and decreased CT densities of the thyroid gland in our patients.

When evaluating whether CT densities correlated with serum TSH concentrations, we found that mean CT densities of the thyroid gland decreased from before to after radiation therapy in $73.9 \%$ of our patients, the median decrease in CT density being $16.8 \mathrm{HU}$, and a significant decrease being defined as $\geq 16.8 \mathrm{HU}$. On the basis of these decreases, we allocated our patients to two groups for analysis: those with a decrease equal to or greater than the median and those with a decrease below the median. Serum TSH concentrations were significantly higher in the former group. Many clinical symptoms of hypothyroidism, such as general malaise, hypohidrosis and dry skin, are non-specific, which makes early detection of hypothyroidism based on clinical symptoms difficult. Subclinical hypothyroidism is recognized as possibly exacerbating hypercholesterolemia, atherosclerosis and myocardial infarction $(14,15)$. Thus, assessment of serum TSH is important for early detection and treatment of subclinical hypothyroidism. When the CT density of the thyroid gland decreases significantly from before to after radiation therapy, serum TSH increases and hypothyroidism may develop.

This study had certain limitations. Because of its retrospective design, the timing of measurement of CT densities and evaluation of thyroid function in relation to timing of radiation therapy was inconsistent. Future studies should ensure for uniform timing of these variables.

In conclusion, significant decreased CT density of the thyroid gland from before to after radiation therapy may be a predictor of hypothyroidism caused by radiation therapy.

\section{Conflicts of Interest}

The Authors declare that no grants were received specifically for this study.

\section{References}

1 Kim MY, Yu T and Wu HG: Dose-volumetric parameters for predicting hypothyroidism after radiotherapy for head and neck cancer. Jpn J Clin Oncol 44: 331-337, 2014.

2 Fujiwara M, Kamikonya N, Odawara S, Suzuki H, Niwa Y, Takada Y, Doi H, Terada T, Uwa N, Sagawa K and Hirota S: The threshold of hypothyroidism after radiation therapy for head and neck cancer: a retrospective analysis of 116 cases. J Radiat Res 56: 577-582, 2015.

3 Ling S, Bhatt AD, Brown NV, Nguyen P, Sipos JA, Chakravarti A and Rong Y: Correlative study of dose to thyroid and incidence of subsequent dysfunction after head and neck radiation. Head Neck 39: 548-554, 2017.

4 Kamijo K: Clinical studies on thyroid CT number in chronic thyroiditis. Endocr J 41: 19-23, 1994. 
5 Pandey V, Reis M and Zhou Y: Correlation Between Computed Tomography Density and Functional Status of the Thyroid Gland. J Comput Assist Tomogr 40: 316-319, 2016.

6 Canaris GJ, Manowitz NR, Mayor G and Ridgway EC: The Colorado thyroid disease prevalence study. Arch Intern Med 160: 526-534, 2000.

7 Alterio D, Jereczek-Fossa BA, Franchi B, D’Onofrio A, Piazzi V, Rondi E, Ciocca M, Gibelli B, Grosso E, Tradati N, Mariani L, Boboc GI and Orecchia R: Thyroid disorders in patients treated with radiotherapy for head-and-neck cancer: a retrospective analysis of seventy-three patients. Int $\mathrm{J}$ Radiat Oncol Biol Phys 67: 144-150, 2007.

8 Norris AA, Amdur RJ, Morris CG and Mendenhall WM: Am J Hypothyroidism when the thyroid is included only in the low neck field during head and neck radiotherapy. Clin Oncol 29: 442-445, 2006.

9 Iida Y, Konishi J, Harioka T, Misaki T, Endo K and Torizuka K: Thyroid CT number and its relationship to iodine concentration. Radiology 147: 793-795, 1983.

10 Imanishi Y, Ehara N, Shinagawa T, Tsujino D, Endoh I, Baba K, Kanemaki Y, Iida S, Hayakawa M, Miyazaki O, Shirakawa K and Nosaka S: Correlation of CT values, iodine concentration, and histological changes in the thyroid. J Comput Assist Tomogr 24: 322-326, 2000.

11 Maldjian PD and Chen T: Is visual assessment of thyroid attenuation on unenhanced CT of the chest useful for detecting hypothyroidism? Clin Radiol 71: 1199.e9-1199.e14, 2016.
12 Bakhshandeh M, Hashemi B, Mahdavi SR, Nikoofar A, Vasheghani M and Kazemnejad A: Normal tissue complication probability modeling of radiation-induced hypothyroidism after head-and-neck radiation therapy. Int J Radiat Oncol Biol Phys 85: 514-521, 2013.

13 Fujiwara Y, Chayahara N, Mukohara T, Kiyota N, Tomioka H, Funakoshi $\mathrm{Y}$ and Minami H: Hypothyroidism in patients with colorectal carcinoma treated with fluoropyrimidines. Oncol Rep 30: 1802-1806, 2013.

14 Series JJ, Biggart EM, O'Reilly DS, Packard CJ and Shepherd $\mathrm{J}$ : Thyroid dysfunction and hypercholesterolaemia in the general population of Glasgow, Scotland. Clin Chim Acta 15: 217-221, 1988.

15 Hak AE, Pols HAP, Visser TJ, Drexhage HA, Hofman A and Witteman JC: Subclinical hypothyroidism is an independent risk factor for atherosclerosis and myocardial infarction in elderly women: the Rotterndam Study. Ann Intern Med 132: 270-278, 2000 .
Received October 27, 2017

Revised November 15, 2017

Accepted November 17, 2017 